
$\$$ Research Square
Preprints are preliminary reports that have not undergone peer review.
They should not be considered conclusive, used to inform clinical practice, or referenced by the media as validated information.

\title{
Application of Chromosome Microarray Analysis and Karyotype Analysis in Diagnostic Assessment of Abnormal Down's Syndrome Screening Results
}

\author{
Han Kang \\ Chengdu Women's and Children's Central Hospital, School of Medicine, University of Electronic Science and Technology of China \\ Lingxi Wang \\ Chengdu Women's and Children's Central Hospital, School of Medicine, University of Electronic Science and Technology of China \\ Xingyu Li \\ Chengdu Women's and Children's Central Hospital, School of Medicine, University of Electronic Science and Technology of China \\ Chonglan Gao \\ Chengdu Women's and Children's Central Hospital, School of Medicine, University of Electronic Science and Technology of China \\ Yamei Xie \\ Chengdu Women's and Children's Central Hospital, School of Medicine, University of Electronic Science and Technology of China \\ Yu Hu ( $\nabla$ tianshuibi@foxmail.com ) \\ Chengdu Women's and Children's Central Hospital, School of Medicine, University of Electronic Science and Technology of China
}

\section{Research Article}

Keywords: Chromosome microarray analysis, karyotype analysis, Down's syndrome screening, prenatal diagnosis

Posted Date: September 13th, 2021

DOI: https://doi.org/10.21203/rs.3.rs-846353/v1

License: (c) (i) This work is licensed under a Creative Commons Attribution 4.0 International License. Read Full License 


\section{Abstract}

Background: Although screening for fetal aneuploidy with the use of cell-free DNA obtained from maternal plasma is highly effective, biomarkers screening is in extensive use in economically underdeveloped areas and poor population. This study aims to explore the application value of chromosomal microarray analysis (CMA) and karyotype analysis in prenatal diagnosis for pregnant women with abnormal Down's syndrome (DS) screening results.

Methods: The study recruited 813 pregnant women with abnormal DS screening results from Chengdu Women's and Children's Central Hospital. They underwent amniocentesis to obtain fetal amniotic fluid for CMA and G-band karyotype analysis. An Affymetrix CytoScan $750 \mathrm{~K}$ Array chip was used for CMA analysis according to the manufacturer's instructions.

Results: In total, CMA identified $21 / 813$ abnormal results, which was more efficient than karyotype analysis(10/813, $\mathrm{P}<0.001$.) CMA is equivalent to traditional karyotype analysis for the prenatal diagnosis of aneuploidies. However, CMA identified $1.60 \%$ more copy number variants(CNVs) than karyotype analysis. These pathogenic/likely pathogenic(P/LP) CNVs ranged from $159 \mathrm{~Kb}$ deletion to $3616 \mathrm{~Kb}$ deletion. $53.8 \%$ of them were recurrent pathogenic $\mathrm{CNV}$ s associated with risk of neurodevelopmental disorders. CMA identified 7 variants of uncertain significance (VUS) results, including 6 microduplication and 1 microdeletion, with the size ranged from $840 \mathrm{~kb}-1484 \mathrm{~kb}$. Karyotype analysis identified 2 mosaic sex chromosome aneuploidy, 2 balanced translocation and 1 mosaic balanced translocation, which could not be identified by CMA.

Conclusions: Performing both CMA and karyotype analysis simultaneously is more beneficial to pregnant women with abnormal DS screening results.

\section{Background}

DS is the most common congenital cause of mental disability and also leads to numerous metabolic and structural problems. Since 1984 Irwin R and colleagues found an association between low maternal serum a-fetoprotein and fetal chromosomal abnormalities [1], several biomarkers have been observed in abnormally high or low concentrations in the serum of pregnant women whose fetuses are affected by DS.[2-5] Although screening for fetal aneuploidy with the use of cfDNA obtained from maternal plasma is highly effective[6], biomarkers screening is in extensive use in economically underdeveloped areas and poor population. When screening tests predict a high risk of DS, an invasive diagnostic test (amniocentesis or chorionic villus sampling) is usually needed to confirm the diagnosis.[7]

Before the era of microarray, G-banded karyotyping was the gold standard diagnostic test for pregnant women whose screening tests predict a high risk. Gbanded karyotyping can identify only $5-10 \%$ deletion and duplications that are $\geq 5 \mathrm{Mb}$.[8] $1.2 \%$ congenital anomalies are caused by pathogenic CNVs, including microdeletions and duplications) while common trisomies (trisomy $21,18,13$ ) accounts for $0.2 \%$. [9] For over a decade, CNV analysis by CMA has been broadly used for detection of genomic imbalances at a much higher resolution than conventional methods such as G-banded karyotyping. It was recommended as a first-tier approach for the prenatal evaluation of fetuses with structural anomalies observed by ultrasound.[10,11] CMA includes array comparative genomic hybridization (aCGH) and single nucleotide polymorphism (SNP) array. Compared with aCGH, SNP array has the advantage of detecting triploidy and regions of homozygosity which might indicate uniparental disomy (UPD).[12]

In this study we summarized the SNP array and karyotype results of 813 cases whose biomarker screening of DS was abnormal.

\section{Methods}

\subsection{Patients}

A total of 813 pregnant women at the Chengdu Women's and Children's Central Hospital, School of Medicine, University of Electronic Science and Technology of China whose second-trimester biochemical markers screening results were abnormal were enrolled in this study from January 2018 to June 2021 . Patients enrolled in the study were aged 18 to 34 years, with an average $28.48 \pm 3.33$ years of age. All pregnant women underwent amniocentesis to obtain fetal amniotic fluid for SNP array and G-band karyotype analysis. This study was approved by the Medical Ethics Committee of Chengdu Women's and Children's Central Hospital and all pregnant women signed informed consent forms.

\subsection{Karyotype analysis and CMA methodology}

Three tubes(10mL*3 tubes) of amniotic fluid was collected under ultrasound-guided localization at 18-25 gestational weeks. Two tubes were used for karyotype analysis, and one tube for CMA. For amniotic fluid samples with maternal cell contamination, the CMA test was performed after the amniotic fluid cells were cultured.

Karyotype analysis was performed independently by two individuals using two cell culture systems. After cell culture and sample preparation, a LABB M9120 instrument (Shanghai Beion Medical Technology, Shanghai, China) and matching image analysis software were used for chromosome karyotype scanning and analysis. At least three cell karyotypes were analyzed for each culture, and 20 karyotypes were counted. For the cases with chromosome mosaicism, more karyotypes were counted or analyzed. Karyotype analysis and descriptions were based on the International Human Cytogenetic Naming System(2016).

Genomic DNA from amniotic fluid was extracted using a QIAamp DNA Blood Mini Kit (QIAGEN, Germany) according to the manufacturer's instructions. An Affymetrix CytoScan $750 \mathrm{~K}$ Array (Affymetrix, USA) chip was used for CMA analysis according to the manufacturer's instructions. ChAS 4.2 software was used for analysis.

\subsection{Results categorization}


The detected results were categorized into aneuploidy and CNVs including gross deletion, gross duplication, microdeletion, microduplication and loss of heterozygosity $(\mathrm{ROH})$. An arbitrary line was drawn at $5 \mathrm{Mb}$ to differentiate between micro and gross deletion and duplication.

\subsection{CNV interpretation and confirmation}

All reported CNVs were based on the National Center for Biotechnology Information human genome build 38. The reported CNVs were classified by five-tiered system according to ACMG TECHNICAL STANDARDS[10] with the assistance of the following databases: the Database of Genomic Variants (DGV, http://dgv.tcag.ca/dgv/), the Online Mendelian Inheritance in Man database (OMIM, http://www.ncbi.nlm.nih.gov/omim), Clinical Genome Resourse(ClinGen, https://www.clinicalgenome.org/), ClinVar( https://www.ncbi.nlm.nih.gov/clinvar/),the Database of Chromosomal Imbalance and Phenotype in Humans using Ensembl Resources (DECIPHER, https://decipher.sanger.ac.uk/), and PubMed (https://www.ncbi.nlm.nih.gov/pubmed/). P, LP and VUS were shown in this study, while likely benign (LB) and benign (B) were not.

\subsection{Inheritance studies}

Inheritance studies were performed using Fluorescence in-situ Hybridization (FISH), conventional karyotyping, or CMA when necessary. For example, unbalanced translocation would need parents' karyotype analysis or FISH depending on CNVs size.

\subsection{Statistical analysis}

SPSS 19.0 software was used for statistical analysis. Comparisons between groups were performed using a $\chi 2$ test, and P< 0.05 was considered statistically significant.

\section{Results}

\subsection{Overall result}

In this study, a total of 813 pregnant women were enrolled in: 660 had a high risk in DS screening, 48 borderline risk, 9 abnormal Multiple of median (MOM), and 96 abnormal DS screening result with ultrasound abnormalities. CMA and karyotype findings were summarized in Table 1. In high risk group CMA identified 16 abnormal results, karyotype analysis identified 8. In borderline risk group, CMA identified 1 abnormal results, karyotype analysis identified none. In abnormal MOM group, none abnormal results were identified by both methods. In abnormal DS screening result/ ultrasound abnormalities group, CMA identified 4 abnormal results, karyotype analysis identified 2. In total, CMA identified 21/813 abnormal results, which was more efficient than karyotype analysis(10/813, $\mathrm{P}<0.001$.)

Table 1 Abnormal results of CMA and karyotype analysis in 813 pregnant women with DS screening abnormalities

\begin{tabular}{|lllll|}
\hline Group & $\mathrm{n}$ & CMA results/case & Karyotype analysis results case & P value \\
\hline High risk & 660 & 16 & 8 & $<0.001$ \\
\hline Borderline risk & 48 & 1 & 0 & $<0.001$ \\
\hline Abnormal MOM & 9 & 0 & 0 & $/$ \\
\hline Abnormal DS screening results with ultrasound abnormalities & 96 & 4 & 2 & $<0.001$ \\
\hline Total & 813 & 21 & 10 & $<0.001$ \\
\hline
\end{tabular}

\subsection{CMA results}

CMA identified 21 abnormal results in total, with the size ranged from $159 \mathrm{~Kb}$ deletion (microdeletion of the $\mathrm{X}$ chromosome, including Duchenne muscular dystrophy (DMD) gene) to $155 \mathrm{Mb}$ whole chromosome gain/loss $(\mathrm{XXX} / \mathrm{X})$. They could be grouped into aneuploidy $(0.84 \%, 7 / 813)$, gross duplication( $0.012 \%$, 1/813), microdeletion(0.98\%, 8/813), microduplication(0.74\%, 5/813).(Table 2,3) Overall, 14 P/LP CNVs were detected in 14 fetuses(Table 3$): 2$ case had a microdeletion in the region of azoospermia factor (AZF) locus of the $Y$ chromosome , 2 case had microdeletion in the $X$ chromosome involving DMD and ichthyosis respectively. 10 cases had an autosomal CNVs including 1 case of $1 \mathrm{q} 21.1$ recurrent microduplication syndrome, 1 case of $2 \mathrm{p} 16.1 \mathrm{p} 15$ microduplication, 1 case of 3q29 microdeletion, 1 case of 11q22.1q23.1 duplication, 1 case of $16 \mathrm{p} 13.11$ recurrent microdeletion syndrome, 1 case of $16 \mathrm{p} 13.11$ recurrent microduplication syndrome, 1 case of recurrent $16 \mathrm{p} 12.1$ microdeletion syndrome, 1 case of $16 \mathrm{p} 11.2$ microduplication syndrome, 1 case of renal cysts and diabetes(RCAD) syndrome, and 1 case of 22q11 duplication syndrome. Among the CNVs mentioned above, only one(11q22.1q23.1 duplication, $9606 \mathrm{~kb}$ ) was identified by karyotype analysis.

CMA identified 7 VUS results, including 6 microduplication and 1 microdeletion, with the size ranged from $840 \mathrm{~kb}-1484 \mathrm{~kb}$. None of them was identified by karyotype analysis. CMA identified 1 ROH(arr[GRCh38] 4q21.22q22.3(83481723_95588274) hmz).

Table 2 The findings of CMA in 813 pregnant women with abnormal DS screening results 


\begin{tabular}{|lll|}
\hline Categorization & $\mathrm{n}$ & $\%$ \\
\hline Aneuploidy & 7 & $0.84 \%$ \\
\hline Gross duplication & 1 & $0.012 \%$ \\
\hline Microdeletion & 8 & $0.98 \%$ \\
\hline Microduplication & 5 & $0.74 \%$ \\
VUS & 7 & $0.84 \%$ \\
\hline Total & 28 & $3.44 \%$ \\
\hline
\end{tabular}

Table 3 P/LP CNVs in 14 fetuses by CMA

\begin{tabular}{|c|c|c|c|c|c|c|c|}
\hline No & CMA results & categorization & $\begin{array}{l}\text { known } \\
\text { syndromes }\end{array}$ & $\begin{array}{l}\text { dosage } \\
\text { sensitive } \\
\text { gene/region }\end{array}$ & $\begin{array}{l}\text { OMIM } \\
\text { gene } \\
\text { count }\end{array}$ & $\begin{array}{l}\text { size of } \\
\text { CNVs/kb }\end{array}$ & Inheritance \\
\hline 1 & $\operatorname{arr}[G R C h 38] Y q 11.223$ 22658726_26274233x0 & $\mathrm{P}$ & AZFc & / & 11 & 3616 & NA \\
\hline 2 & $\operatorname{arr}[G R C h 38]$ Yq11.223 (24889425_28231736)x0 & $\mathrm{P}$ & AZFc & / & 11 & 3342 & father \\
\hline 3 & arr[GRCh38]Xp21.1 (31809962_31968905)x0 & $P$ & / & DMD & 1 & 159 & mother \\
\hline 4 & arr[GRCh38]Xp22.31 6537109_8167604)x1 & $\mathrm{P}$ & STS & $\begin{array}{l}\text { STS/Xp22.31 } \\
\text { recurrent } \\
\text { region }\end{array}$ & 4 & 1630 & NA \\
\hline 5 & $\operatorname{arr}[$ GRCh38]1q21.1q21.2(147053151_148360058)x3 & $\mathrm{P}$ & $\begin{array}{l}\text { 1q21.1 recurrent } \\
\text { microduplication }\end{array}$ & $\begin{array}{l}1 \mathrm{q} 21.1 \\
\text { recurrent } \\
\text { region }\end{array}$ & 9 & 1812 & NA \\
\hline 6 & $\operatorname{arr}[$ GRCh38]2p16.1p15(60148343_61784764)x3 & LP & / & / & 7 & 1636 & de novo \\
\hline 7 & arr[GRCh38]3q29 (193373606_195885016)x1 & LP & / & / & 16 & 2511 & NA \\
\hline 8 & $\begin{array}{l}\text { arr[GRCh38]11q22.1q23.1 } \\
\text { 102192300_111795977)×3 }\end{array}$ & LP & / & / & 50 & 9606 & de novo \\
\hline 9 & $\operatorname{arr}[$ GRCh38]16p13.11 (14799119_16364567)x1 & $\mathrm{P}$ & $\begin{array}{l}16 \mathrm{p} 13.11 \\
\text { recurrent } \\
\text { microdeletion }\end{array}$ & $\begin{array}{l}16 \mathrm{p} 13.11 \\
\text { recurrent } \\
\text { region }\end{array}$ & 14 & 1565 & $\begin{array}{l}\text { mother(learning } \\
\text { disorder) }\end{array}$ \\
\hline 10 & $\begin{array}{l}\operatorname{arr}[\text { GRCh38]16p13.11p12.3 } \\
\left(15225421 \_18148856\right) \times 3\end{array}$ & $\mathrm{P}$ & $\begin{array}{l}16 \mathrm{p} 13.11 \\
\text { recurrent } \\
\text { microduplication }\end{array}$ & $\begin{array}{l}16 \mathrm{p} 13.11 \\
\text { recurrent } \\
\text { region }\end{array}$ & 10 & 2923 & mother \\
\hline 11 & arr[GRCh38]16p12.1 (21728879_22430686)x1 & $\mathrm{p}$ & $\begin{array}{l}\text { Recurrent } \\
16 \mathrm{p} 12.1 \\
\text { microdeletion }\end{array}$ & / & 5 & 702 & father \\
\hline 12 & 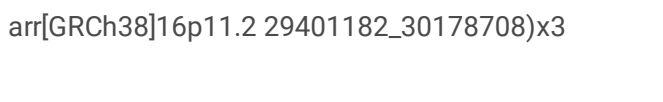 & $\mathrm{P}$ & $\begin{array}{l}16 \mathrm{p} 11.2 \\
\text { microduplication } \\
\text { syndrome }\end{array}$ & $\begin{array}{l}16 \mathrm{p} 11.2 \\
\text { recurrent } \\
\text { region }\end{array}$ & 26 & 778 & father \\
\hline 13 & $\operatorname{arr}[$ GRCh38]17q12(36466620_37940921)x1 & $\mathrm{P}$ & RCAD syndrome & $\begin{array}{l}\text { HNF1B/17q12 } \\
\text { recurrent } \\
\text { (RCAD } \\
\text { syndrome) } \\
\text { region }\end{array}$ & 14 & 1474 & NA \\
\hline 14 & $\operatorname{arr}[$ GRCh38]22q11.21(18153983_21110475)x3 & $\mathrm{P}$ & $\begin{array}{l}\text { 22q11 } \\
\text { duplication } \\
\text { syndrome }\end{array}$ & / & 45 & 2956 & mother \\
\hline
\end{tabular}

DMD: Duchenne muscular dystrophy, STS: Steroid sulphatase deficiency, RCAD: renal cysts and diabetes, HNF1B: hepatocyte nuclear factor 1beta.

\subsection{Karyotype results}

Traditional karyotype identified 10/813 abnormal results: including 4 trisomy 21(No 1-4), 2 superfemale syndrome(No 5-6), 1 Tunner syndrome(No 7), 2 mosaic sex chromosome aneuploidy( No 8-9),1 gross duplication(No10). Besides, 2 balanced translocation(No 11-12) and 1 mosaic balanced translocation(No 13) were identified. CMA identified all of these aneuploidies. However, CMA identified neither of the two mosaic sex chromosome aneuploidy for their low proportion(<=10\%). Both of the balanced translocation were inherited from healthy father, and normal results of CMA also suggested they were truly balanced. For the mosaic balanced translocation, although CMA result was normal, we couldn't discriminate it between truly balanced and unbalance.

Table 4 The findings of karyotype analysis in 813 pregnant women with abnormal DS screening results 


\begin{tabular}{llll} 
No & karyotype results & Known syndromes & Inheritance \\
\hline $1-3$ & $47,-,+21$ & Down's syndrome & de novo \\
\hline 4 & $46,-, \operatorname{rob}(14 ; 21)(\mathrm{q} 10 \mathrm{q} 10),+21$ & Down's syndrome & NA \\
\hline $5-6$ & $47, \mathrm{XXX}$ & Superfemale syndrome & de novo \\
\hline 7 & $45, \mathrm{X}$ & Tunner syndrome & de novo \\
\hline 8 & $\operatorname{mos} 45, \mathrm{X}[7] / 46, \mathrm{XX}[127]$ & Tunner syndrome(mosaic) & de novo \\
\hline 9 & $\operatorname{mos} 45, \mathrm{X}[15] / 46, \mathrm{XY}[135]$ & Tunner syndrome/Hermaphroditism & de novo \\
\hline 10 & $46,-, \operatorname{dup}(11)(\mathrm{q} 22.2 q 23.1)$ & $/$ & de novo \\
\hline 11 & $45,-, \operatorname{rob}(14 ; 22)(\mathrm{q} 10 ; q 10)$ & $/$ & Inherited from normal father \\
\hline 12 & $46,-, \mathrm{t}(2 ; 20)(\mathrm{p} 23 ; q 13.1)$ & $/$ & Inherited from normal father \\
\hline 13 & $\operatorname{mos} 46,-, \mathrm{t}(3 ; 6)(\mathrm{q} 11.2 ; q 25)[9] / 46,-[43]$ & $/$ & de novo
\end{tabular}

\section{Discussion}

CMA, also known as molecular karyotyping, has gradually replaced conventional G-banded karyotyping as the first-tier diagnostic test for the individual with developmental delay, mental retardation, autism spectrum disorder, and/or multiple congenital anomalies, as well as for prenatal evaluation of fetuses with structural anomalies observed by ultrasound.[10] Compared with karyotype analysis, CMA is capable of detecting clinically significant submicroscopic aberrations up to a few kb. SNP array uses high-density oligonucleotide-based arrays in which target probes are chosen from DNA locations known to vary between individuals by a single base pair. CNVs are determined by measuring the absolute fluorescence probe intensities of the patient sample compared with the intensities of multiple normal controls.[13] In this study, we used CMA(SNP array platform) and karyotype analysis for prenatal diagnosis of pregnant women with abnormal DS screening results. CMA is equivalent to traditional karyotype analysis for the prenatal diagnosis of aneuploidies. CMA provided additional clinically relevant information in 13 of pregnancies. CMA could detect $1.60 \%$ more CNVs than karyotype analysis, which is consistent with previous reports.[14] [15] However, the prevalence of P/LP CNVs in DS screening abnormal fetus(1.6\%) is a bit higher than that in common population(1.2\%).[9] Besides, we identified a mendelian monogenetic disease, that a male fetus had a microdeletion in DMD gene. Inheritance studies revealed the abnormalities was inherited from his mother who had no family history.

Among the P/LP CNVs identified by CMA, 53.8\% were recurrent pathogenic CNVs associated with risk of neurodevelopmental disorders. The penetrance for these recurrent pathogenic CNVs vary from race to race, $[16,17]$ and there was no large penetrance data available in Chinese population. So it was difficult to determine the clinical significance of these recurrent pathogenic CNVs, which would cause significant stress to pregnant women and their families, in some cases even resulted in the unnecessary abortion. According to Rosenfeld JA and colleagues[17], higher penetrance is seen with CNVs that have higher de novo frequencies. It was also reported that a strong association between IQ and the probability at which CNV deletions occur de novo.[18] Therefore inheritance studies of parents would be helpful to help determining source and counseling. Among the 7 recurrent pathogenic CNVs detected in this study, 2 refused inheritance studies and chose to terminate the pregnancy, 1 were inherited from mother having learning disorder(de novo) and chose to terminate the pregnancy, and the rest 4 were inherited from healthy parents and chose to continue the pregnancy. We would keep a long-time follow-up for the 4 parents. CMA identified 7 VUS, which is a difficult problem to genetic counseling. Inheritance studies of parents should be performed to help determining source and counseling. Pregnant women and their families should be fully informed of the possible outcomes and provide consent before CMA is performed.

The American College of Obstetrics and Gynecology (ACOG) and the American Maternal-Fetal Medicine Association's 2016 guidelines clearly suggest CMA as a first-line prenatal diagnostic method in pregnant women with ultrasounds structural abnormalities.[11, 19] However, only a few reports had mentioned the effectiveness of CMA in pregnant women with DS screening abnormals. In this study, CMA idenitified $1.60 \%$ more P/LP CNVs than karyotype analysis, which is the first cause of congenital abnormies[9]. So it is necessary to perform CMA in pregnant women with DS screening abnormals.

Despite the advantages of superior sensitivity and faster turn-around time, there are also some disadvantages compared to conventional karyotyping. CMA is unable to detect balanced chromosomal aberrations and mosaic chromosome abnormalities in low proportion. In this study, karyotype analysis identified 2 mosaic sex chromosome aneuploidy (45,X[7]/46,XX[127], 45,X[15]/46,XY[135]). CMA failed to identify both of them. Although the proportions of abnormal cells were low, they might result in some symptoms of Tunner syndrome or hermaphroditism according to previous reports[20,21] and our experience in adults with such karyotype. CMA can detect trisomy 13/21 but cannot discern whether it resulted from a non-disjunction event or due to a translocation. In such cases, karyotype analysis of the fetus and the parents is essential for determining reproductive risk for future offspring. Besides, karyotype analysis provided additional clinically relevant information in 3 of pregnancies, 2 balanced translocation and 1 mosaic balanced translocation, which would be helpful for future pregnancies.

\section{Conclusions}

In total, CMA identified 21/813 abnormal results, which was more efficient than karyotype analysis (10/813, P<0.001.) CMA identified 13 more definite CNVs than karyotype analysis. However, karyotype analysis identified 2 mosaic sex chromosome aneuploidy, 2 balanced translocation and 1 mosaic balanced translocation, which could not be identified by CMA despite its advantages of superior sensitivity and faster turn-around time.

In conclusion, performing both CMA and karyotype analysis simultaneously is more beneficial to pregnant women with abnormal DS screening results. 


\section{Abbreviations}

CMA: chromosomal microarray analysis; DS: Down's syndrome; CNVs: copy number variants; P/LP: pathogenic/likely pathogenic; VUS: variants of uncertain significance; aCGH: array comparative genomic hybridization; SNP: single nucleotide polymorphism; UPD: uniparental disomy; ROH: loss of heterozygosity; DGV:Database of Genomic Variants; OMIM: the Online Mendelian Inheritance in Man database ; ClinGen:Clinical Genome Resourse; DECIPHER: the Database of Chromosomal Imbalance and Phenotype in Humans using Ensembl Resources; FISH: Fluorescence in-situ Hybridization; MOM: Multiple of median; DMD: Duchenne muscular dystrophy; AZF: azoospermia factor; RCAD: renal cysts and diabetes; ACOG: The American College of Obstetrics and Gynecology.

\section{Declarations}

\section{Ethics approval and consent to participate}

This study was performed in accordance with the Declaration of Helsinki and was approved by the Medical Ethics Committee of Chengdu Women's and Children's Central Hospital and all pregnant women signed informed consent forms.

\section{Consent for publication}

Not applicable.

\section{Availability of data and materials}

The data that support the findings of this study are available on request from the corresponding author.

\section{Competing interests}

The authors declare that they have no competing interests.

\section{Funding}

No grants or external funding to report.

\section{Authors' contributions}

Y.H initiated the study and drafted the manuscript; H.K designed the study and provided input into analysis design; H.K, LX.W, XY.L and YM.X performed laboratory work, CL.G and Y.H performed the clinical work. All authors contributed significantly, read and approved the final manuscript.

\section{Acknowledgements}

Not applicable

\section{References}

1. Merkatz IR, et al, An association between low maternal serum alpha-fetoprotein and fetal chromosomal abnormalities. Am J Obstet Gynecol, 1984. 148(7): p. 886-94.

2. Liu Y, et al, Diagnostic value of ultrasonographic combining biochemical markers for Down syndrome screening in first trimester: a meta-analysis. Prenat Diagn, 2015. 35(9): p. 879-87.

3. Spencer K., Screening for Down syndrome. Scand J Clin Lab Invest Suppl, 2014. 244: p. 41-7.

4. Alldred SK., et al, First trimester serum tests for Down's syndrome screening. Cochrane Database Syst Rev, 2015(11): p. CD011975.

5. Casals E, et al, First-trimester biochemical markers for Down syndrome. Prenat Diagn, 1999. 19(1): p. 8-11.

6. Norton ME., et al, Cell-free DNA analysis for noninvasive examination of trisomy. N Engl J Med, 2015. 372(17): p. $1589-97$.

7. ACOG Practice Bulletin No. 77: screening for fetal chromosomal abnormalities. Obstet Gynecol, 2007. 109(1): p. $217-27$.

8. Shaffer LG, American College of Medical Genetics guideline on the cytogenetic evaluation of the individual with developmental delay or mental retardation. Genet Med, 2005. 7(9): p. 650-4.

9. Evans MI, RJ Wapner and RL Berkowitz, Noninvasive prenatal screening or advanced diagnostic testing: caveat emptor. Am J Obstet Gynecol, 2016. 215(3): p. 298-305.

10. Riggs ER, et al, Technical standards for the interpretation and reporting of constitutional copy-number variants: a joint consensus recommendation of the American College of Medical Genetics and Genomics (ACMG) and the Clinical Genome Resource (ClinGen). Genet Med, 2020. $22(2)$ : p. $245-257$.

11. Committee Opinion No. 581: the use of chromosomal microarray analysis in prenatal diagnosis. Obstet Gynecol, 2013. 122(6): p. 1374-7.

12. Del GD, et al, Diagnostic testing for uniparental disomy: a points to consider statement from the American College of Medical Genetics and Genomics (ACMG). Genet Med, 2020. 22(7): p. 1133-1141.

13. Zhang Y, M Zhong and D Zheng, Chromosomal mosaicism detected by karyotyping and chromosomal microarray analysis in prenatal diagnosis. J Cell Mol Med, 2021. 25(1): p. 358-366.

14. Wapner RJ, et al, Chromosomal microarray versus karyotyping for prenatal diagnosis. N Engl J Med, 2012. 367(23): p. $2175-84$. 
15. Hu R, et al, [Application of Array-based Comparative Genomic Hybridization in Diagnostic Assessment of Abnormal Prenatal Serological Screening Results of Down's Syndrome]. Sichuan Da Xue Xue Bao Yi Xue Ban, 2021. 52(2): p. 319-325.

16. Kendall KM, et al, Cognitive performance and functional outcomes of carriers of pathogenic copy number variants: analysis of the UK Biobank. Br $\mathrm{J}$ Psychiatry, 2019. 214(5): p. 297-304.

17. Rosenfeld JA, et al, Estimates of penetrance for recurrent pathogenic copy-number variations. Genet Med, 2013. 15(6): p. 478-81.

18. Jacquemont S, et al, Mirror extreme BMI phenotypes associated with gene dosage at the chromosome 16p11.2 locus. Nature, 2011. 478(7367): p. 97102.

19. Dugoff L, ME Norton and JA Kuller, The use of chromosomal microarray for prenatal diagnosis. Am J Obstet Gynecol, 2016. 215(4): p. B2-9.

20. Tosson H, SR Rose and LA Gartner, Children with 45,X/46,XY karyotype from birth to adult height. Horm Res Paediatr, 2010. 74(3): p. 190-200.

21. Tosson H, SR Rose and LA Gartner, Description of children with 45,X/46,XY karyotype. Eur J Pediatr, 2012. 171(3): p. 521-9. 